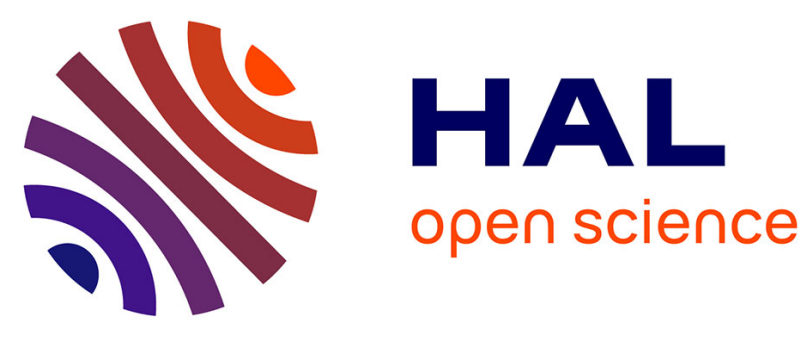

\title{
Stick-slip instabilities in the shear flow of magnetorheological suspensions
}

Modesto Lopez-Lopez, Pavel Kuzhir, Laura Rodriguez Arco, Jaime Caballero Fernandez, Juan D. G. Durán, Georges Bossis

\section{- To cite this version:}

Modesto Lopez-Lopez, Pavel Kuzhir, Laura Rodriguez Arco, Jaime Caballero Fernandez, Juan D. G. Durán, et al.. Stick-slip instabilities in the shear flow of magnetorheological suspensions. Journal of Rheology, 2013, 57 (4), pp.1101. 10.1122/1.4804358 . hal-00846054

\section{HAL Id: hal-00846054 https://hal.science/hal-00846054}

Submitted on 19 Jul 2013

HAL is a multi-disciplinary open access archive for the deposit and dissemination of scientific research documents, whether they are published or not. The documents may come from teaching and research institutions in France or abroad, or from public or private research centers.
L'archive ouverte pluridisciplinaire HAL, est destinée au dépôt et à la diffusion de documents scientifiques de niveau recherche, publiés ou non, émanant des établissements d'enseignement et de recherche français ou étrangers, des laboratoires publics ou privés. 


\title{
Stick-slip instabilities in the shear flow of magnetorheological suspensions
}

\author{
Modesto T. López-López, ${ }^{1}$ Pavel Kuzhir, ${ }^{2}$ Laura Rodríguez -Arco ${ }^{1}$, \\ Jaime Caballero-Hernández, ${ }^{1}$ Juan D. G. Durán, ${ }^{1}$ and Georges Bossis ${ }^{2}$ \\ ${ }^{I}$ Departamento de Física Aplicada, Facultad de Ciencias, Universidad de Granada, Campus de Fuentenueva, \\ 18071 Granada, Spain \\ ${ }^{2}$ Université de Nice Sophia Antipolis, Laboratoire de Physique de la Matière Condensée, CNRS UMR 7336, \\ Parc Valrose, 06108 Nice cedex 2, France
}

\section{Synopsys}

This work is devoted to the stick-slip instabilities that appear in the shear flow of highly concentrated suspensions of magnetic microparticles. The effect of the applied magnetic field strength was analyzed in details. With this aim, homogeneous suspensions of iron microparticles with concentration near the limit of maximum-packing fraction were prepared, and shear-flow measurements were performed in a controlled-rate mode using a rheometer provided with a rough parallel-plate geometry. For each given value of the shear rate, the time evolution of the shear stress was monitored for at least $20 \mathrm{~min}$. Saw-tooth-like stress oscillations, typical of stick-slip instabilities, were obtained at low enough shear rate values. The measurements were restricted to small enough oscillations, at which the rheometer was still able to maintain the shear rate constant. From the microscopic viewpoint, these stick-slip instabilities principally appear due to the periodic failure and healing of the field-induced particle structures, as inferred from experimental observations. This hypothesis is corroborated by a theoretical model developed on the basis of the balance of the magnetic and hydrodynamic torques over the particle structures, allows us to predict the correct order of magnitude of the main parameters of the stick-slip instabilities, including the amplitude and period of the stress oscillations. 


\section{INTRODUCTION}

The behavior of yield stress fluids in the vicinity of the yield point has recently attracted much attention in literature. Most of the macroscopic measurements performed on various complex fluids exhibiting a yield stress (concentrated colloidal and granular suspensions, emulsions and micelle solutions, polymer liquids and polymer blends) have shown that a stable steady-state flow is impossible for these fluids at small enough shear rates [Bécu et al. (2006); Bonn et al. (2002); Coussot et al. (2002); Da Cruz et al. (2002); Huang et al. (2005); Jarny et al. (2005); Ovarlez et al. (2006); Ragouilliaux et al. (2007); Rodts et al. (2005); Varadan and Solomon (2003)]. In most cases, the flow curves of these fluids possess an initial section where the stress either decreases or remains quasi-constant with the shear rate - the section where flow instabilities occur. These instabilities are manifested through a loss in the homogeneity of the flow and the deformation fields, which, in many cases, is accompanied by oscillations of the shear stress or shear rate.

From the microscopic point of view, slow shear flows are often accompanied with shear banding and/or phase separation phenomena, as revealed by dynamic light scattering and magnetic resonance imaging for many types of complex fluids [Coussot et al. (2002); Coussot et al. (2005); Fall et al. (2009); Huang et al. (2005); Ianni et al. (2008); Ovarlez et al. (2009)]. According to these works, in the vicinity of the yield point two distinct flow regions coexist: a non-sheared band, containing a high concentration of solid phase, and a highly sheared band, containing predominantly liquid phase. In addition to shear banding, periodic oscillations in the flow behavior at subcritical shear rates have also been reported [Ianni et al. (2008)]. In the phenomenological model of [Picard et al. (2002)], these oscillations were attributed to periodic changes in the suspension fluidity (local relaxation of the stress) within 
the unstable flow region at the decreasing branch of the flow curve. The authors used the term "stick-slip" for these oscillations even though solid friction does not appear explicitly in their theory. From all these previous works, it is clear that the microscopic and macroscopic behaviors of yield stress fluids must strongly depend on the types of interactions in the fluids, and investigations in this field are intensively continued.

Some well-known yield stress fluids for which the flow instabilities have been scarcely investigated are electrorheological (ER) and magnetorheological (MR) suspensions. In these fluids, interparticle interactions can be tuned over several decades by external electric and magnetic fields, respectively. One of the pioneer studies concerning flow irregularities in these fluids was carried out by Klingenberg and Zukosky (1990), who visualized the shear flow of ER suspensions under electric fields applied perpendicular to the rheometer plates. These authors reported the existence of gap-spanning particle aggregates that were continuously ruptured by the shear forces and subsequently reformed by association with the broken parts of the neighboring aggregates. This process is schematized in Fig. 1a. Such periodic failure and reformation of the structures was later reproduced by particle level simulations [Klingenberg et al. (1991)]. Bonnecaze and Brady (1992) also performed simulations of the structure and the stress level in sheared ER fluids and found some dynamics reminiscent of the stick-slip motion at small shear rates (or rather small Mason numbers): a slow deformation regime, characterized by the storage of the electrostatic energy, followed by a rapid release of the energy through viscous dissipation once the aggregates are ruptured, followed by reformation of the aggregates through associations among broken parts, as illustrated in Fig. 1b. Both works by Klingenberg et al. (1991) and by Bonnecaze and Brady (1992) predicted rather irregular stress vs. time responses, with multiple events in different time scales. 
The only work reporting explicit experimental stick-slip phenomena in ER suspensions is, to our knowledge, a short communication by Woestman (1993). This author measured the strain response of ER fluids to applied stresses using a concentric cylinder cell. He found that the inner cylinder turned by short jumps (slip) followed by long periods without distinguishable motion (stick). The resulting stress vs. strain curves showed quite regular oscillations with a sharp increase of the stress followed by a smooth decrease. Apparently, the imposed stress was not kept constant but depended on the inertia and stiffness of the measuring system. Therefore, the quantitative analysis of the observed phenomenon should be taken with care, while the qualitative interpretation in terms of rupture/reformation remains reasonable.

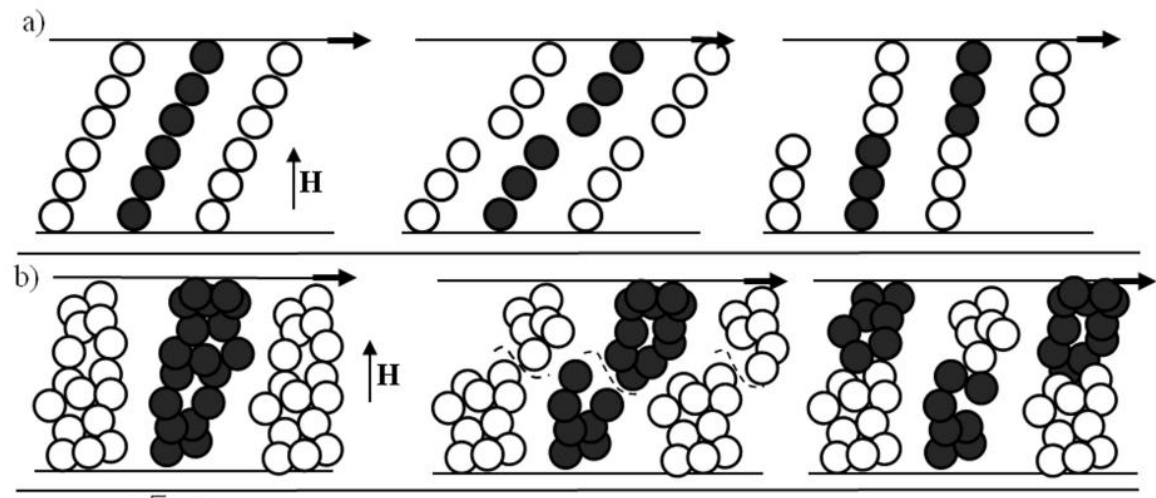

c)
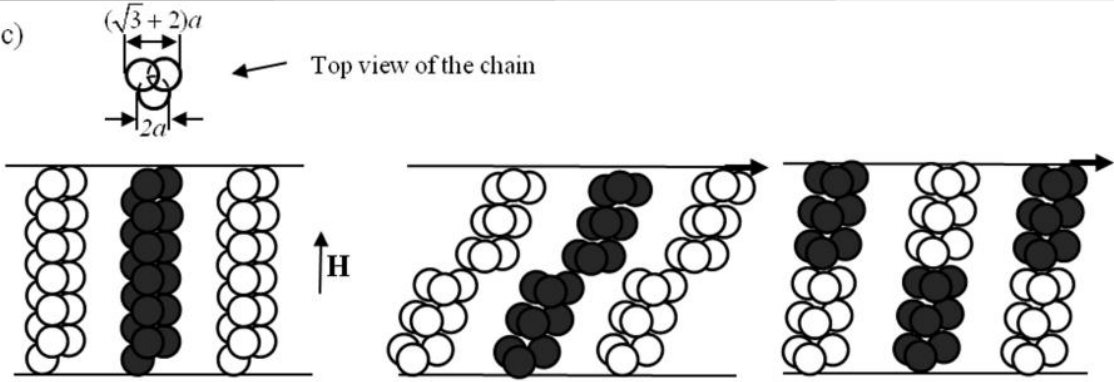

FIG. 1. Periodic rupture/reformation processes that may explain the stick-slip phenomenon, for different types of MR structures: (a) single chains of Klingenberg and Zukosky (1990); (b) thick aggregates used in the particle level simulations of Bonnecaze and Brady (1992); (c) triple-chain aggregates used in the present work. Dashed curves in (b) denote rupture locations. 
In what concerns MR suspensions, the stick-slip instability has been recently reported for concentrated MR fluids exhibiting a shear thickening behavior [Jiang et al. (2012)]. As inferred from this short communication, the authors insist on a frictional scenario to explain the appearance of the stick-slip. They state that the structure evolution of the MR fluid under low shearing should alter micro-gaps between MR particles, and the lubrication performance of the suspending liquid is controlled by the external magnetic field, resulting in periodic transitions between boundary lubrication and hydrodynamic lubrication regimes. Such an interpretation is somewhat different from the above considered scenario of periodically breaking and reforming structures. More information about the role of friction forces on the rheology of ER and MR fluids can be found in [de Vicente and Ramírez (2007), Kuzhir et al. (2009), Tian et al. (2010-a, 2010-b, 2011), Jiang et al. (2011)].

In view of the lack of experimental studies and of the absence of a common interpretation of the stick-slip phenomenon in MR suspensions, we have aimed to conduct a detailed study on stick-slip instabilities. In this manuscript, we focus on the stress response of highly concentrated MR suspensions subjected to imposed shear rates. Our measurements are restricted to relatively small oscillations, at which the rheometer is still able to maintain the imposed shear rate. The experimental oscillating stress signal will be qualitatively compared with the predictions of the simulations of Klingenberg et al. (1991) and Bonnecaze and Brady (1992). In addition, we will present our own theoretical model that, as will be shown, predicts the main features of the stick-slip instabilities observed in our experiments. Finally, we shall try to estimate whether the flow of our suspension corresponds to lubricated contacts between particles (lubricated regime) or to direct contacts with solid friction (frictional regime). 


\section{MATERIALS AND METHODS}

Carbonyl iron powder (Fe-CC, BASF, Germany), was used as solid phase for the preparation of the MR suspensions. According to the manufacturer, these particles have median diameter of $5 \mu \mathrm{m}$. Mineral oil (density and viscosity at $25^{\circ} \mathrm{C}: 0.85 \mathrm{~g} \cdot \mathrm{cm}^{-3}$ and $39.58 \pm$ $0.16 \mathrm{mPa} \cdot \mathrm{s}$, respectively) purchased from Sigma Aldrich (Germany) was used as carrier liquid. MR suspensions with $50 \mathrm{vol} . \%$ approximate concentration were prepared following the protocol described by López-López et al. (2012). The suspension magnetization was measured using a hysteresimeter (S2IS, France), and the magnetization curve is plotted in Fig.2. As is seen in this figure, the suspension remnant magnetization is low as compared to the saturation magnetization $\left(M_{r}=6 \pm 4 \mathrm{kA} / \mathrm{m}\right.$ against $\left.M_{S}=760 \pm 10 \mathrm{kA} / \mathrm{m}\right)$, and the magnetization curve appears to be quasi-linear at magnetic fields, $H<25 \mathrm{kA} / \mathrm{m}$, with the initial magnetic susceptibility being equal to $\chi_{i}=7.2 \pm 0.3$. The whole magnetization curve is satisfactory fitted by a Fröhlich-Kennelly law [Jiles (1991)]: $M=\chi_{i} M_{S} H /\left(\chi_{i} H+M_{S}\right)$.

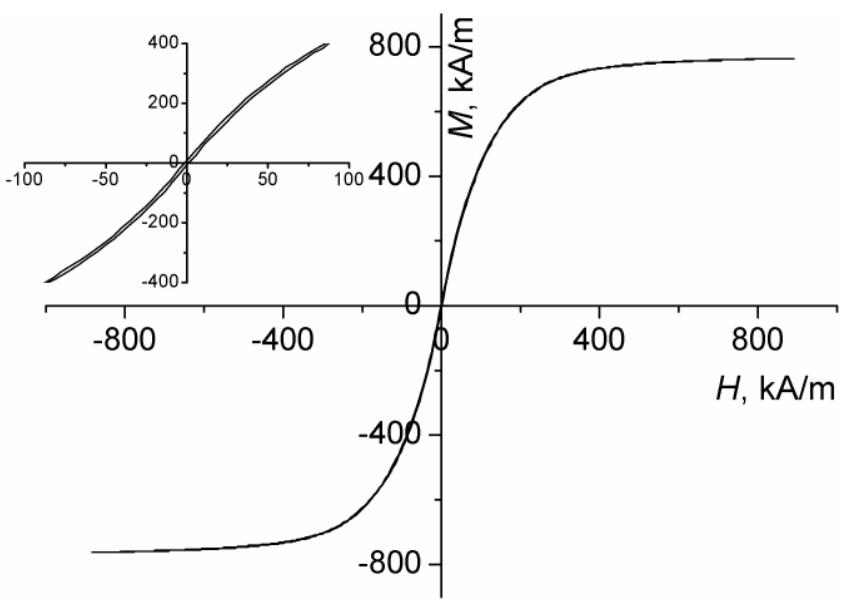

FIG. 2. Magnetization curve of the MR suspension of volume fraction of 50\%. The inset shows the same curve at lower values of the magnetic field.

Rheological measurements were performed at $25{ }^{\circ} \mathrm{C}$ using a rheometer MCR 300 (Physica-Anton Paar). The measuring geometry consisted of a homemade set of non-magnetic 
parallel plates of $20 \mathrm{~mm}$ in diameter, with rough surfaces in order to avoid wall slip. The gap thickness between the lower (stationary) plate and the upper (rotating) plate was $0.35 \mathrm{~mm}$. The applied magnetic field was generated with the help of a solenoid, placed co-axially with the measuring geometry, the technical details being described in Kuzhir et al. (2008).

The experimental protocol used for the rheological measurements was as follows. Prior to the measurements, the MR sample was intensively stirred by using a mechanical vortex mixer. Immediately afterwards, the sample was placed in the measuring system of the rheometer and subjected to a linear shear rate ramp of 1 min of duration in the range $0.1-100$ $\mathrm{s}^{-1}$. Then, a magnetic field of a given intensity was applied and the suspension was left at rest for $30 \mathrm{~s}$. Finally, a constant shear rate (within the range $0.01-200 \mathrm{~s}^{-1}$ ) was applied during the time required to achieve shear strains, $\gamma \gg 1$, and the stress response was measured during this time. Once the measurement at a given shear rate was accomplished, the whole process (starting at the pre-shear stage at zero magnetic field) was repeated at another shear rate. In this way, each point of the shear stress-vs.-shear rate curve was obtained through a long time measurement independent on the suspension history at previous shear rates. Presumably, isotropic structures should constitute the MR suspension before the application of the magnetic field at each step. As will be shown and discussed below, at low enough shear rates the shear stress did not reach a steady state value even after 20 min of shearing at constant shear rate, but instead experienced some regular oscillations with shape very similar to that of the stick-slip motion of a solid body on a solid substrate. If this was the case, we took the mean value of the stress (within its oscillations) as the representative stress for the corresponding shear rate. At this point, it is important to note that the electronic commutation motor of the rheometer Physica MCR 300 does not allow a perfect control of the strain (or shear rate) for the large and rapid variations of the stress signal exhibited by some materials. 
We checked that, at small stress oscillations, the strain signal was almost linear with time. Consequently, we restricted our measurements to relatively weak stress oscillations.

\section{EXPERIMENTAL OBSERVATIONS}

The rheograms of the MR suspensions obtained for four different magnetic fields, using the protocol described in Sec. II, are plotted in Fig. 3. For all the magnetic fields, except zero field, the rheograms show a minimum at some non-zero shear rate. A very similar nonmonotonic stress-shear rate behavior was observed by Pignon et al. (1996) for Brownian suspensions of laponite particles. This shape of the rheogram suggests unstable flows of the suspension, since it is well known that a steady state homogeneous flow with a linear velocity profile is absolutely unstable within the range of shear rates corresponding to the decreasing branch of the rheogram -see for instance Quemada (1982). Within this domain of negative differential viscosity, the shear rate may vary from point to point in the suspension, and, strictly speaking, we are not allowed to define the shear rate in the common way as the ratio of the upper plate speed $v$ to the gap thickness $h$. Therefore, from now in this work the quantity $v / h$ is referred to as the apparent or global shear rate. Note that in the absence of field, we do not observe a clear minimum but the flow curve shows a flat trend at low shear rates, followed by an increase at higher shear rates. As is seen from Fig.3, the flow curves are shifted upwards to higher values of the shear stress when the magnetic field is increased. This is the signature of the classical magnetorheological effect - field induced enhancement of the viscoelastic properties of MR suspensions. What is more important for the present study is that the flow curve minimum becomes much deeper with an increasing magnetic field. This suggests that eventual flow instabilities in MR suspensions are governed principally by magnetic interactions. 


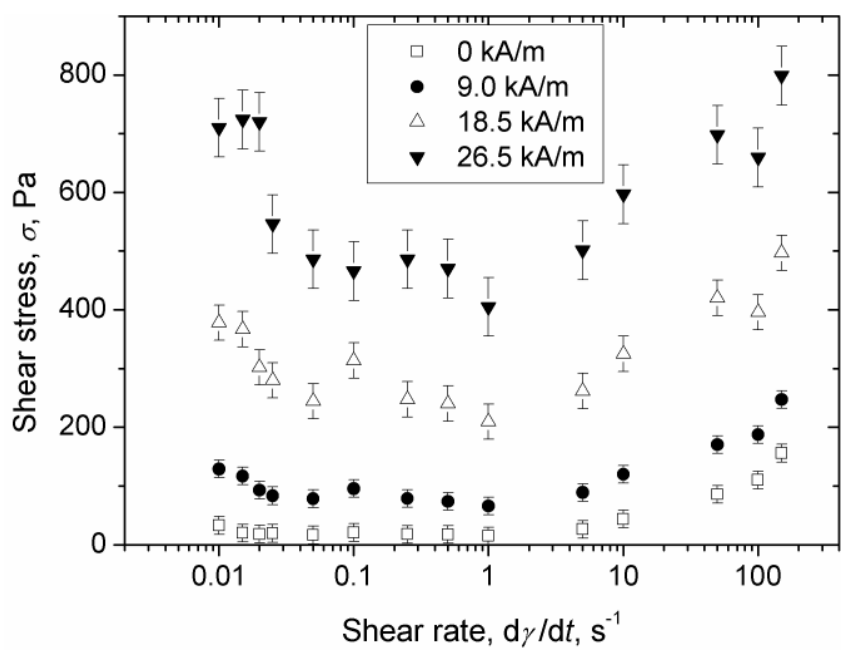

FIG. 3. Experimental flow curves of highly concentrated MR suspension in a semi-log scale for four different applied magnetic fields.

In order to inspect the stability of the MR fluid shear flow at the decreasing branch of the flow curves, we measured the temporal evolution of the stress signal in response to the applied constant shear rate. Typical stress versus time dependences are shown in Fig. 4 for four different values of the applied field, $H_{0}$, and three different values of the applied shear rate, $\dot{\gamma}$. As observed in this figure, at low enough values of $\dot{\gamma}$, the shear stress first increases slowly with time and then, after 200-400 s, a regime characterized by regular stress oscillations with a constant amplitude is reached. These stress oscillations present a sawtooth-like shape with well-defined period and amplitude for shear rates lower than some critical value depending on the applied magnetic field, as illustrated in Fig. $4 \mathrm{~b}$ for $\dot{\gamma}=0.01 \mathrm{~s}^{-1}$. This stress signal is very similar to that inherent of the dry stick-slip motion and has been previously found for concentrated colloidal laponite gels [Pignon et al. (1996)]. The stick process is associated to the increase of the shear stress, while the slip one is characterized by the decrease of this magnitude. 

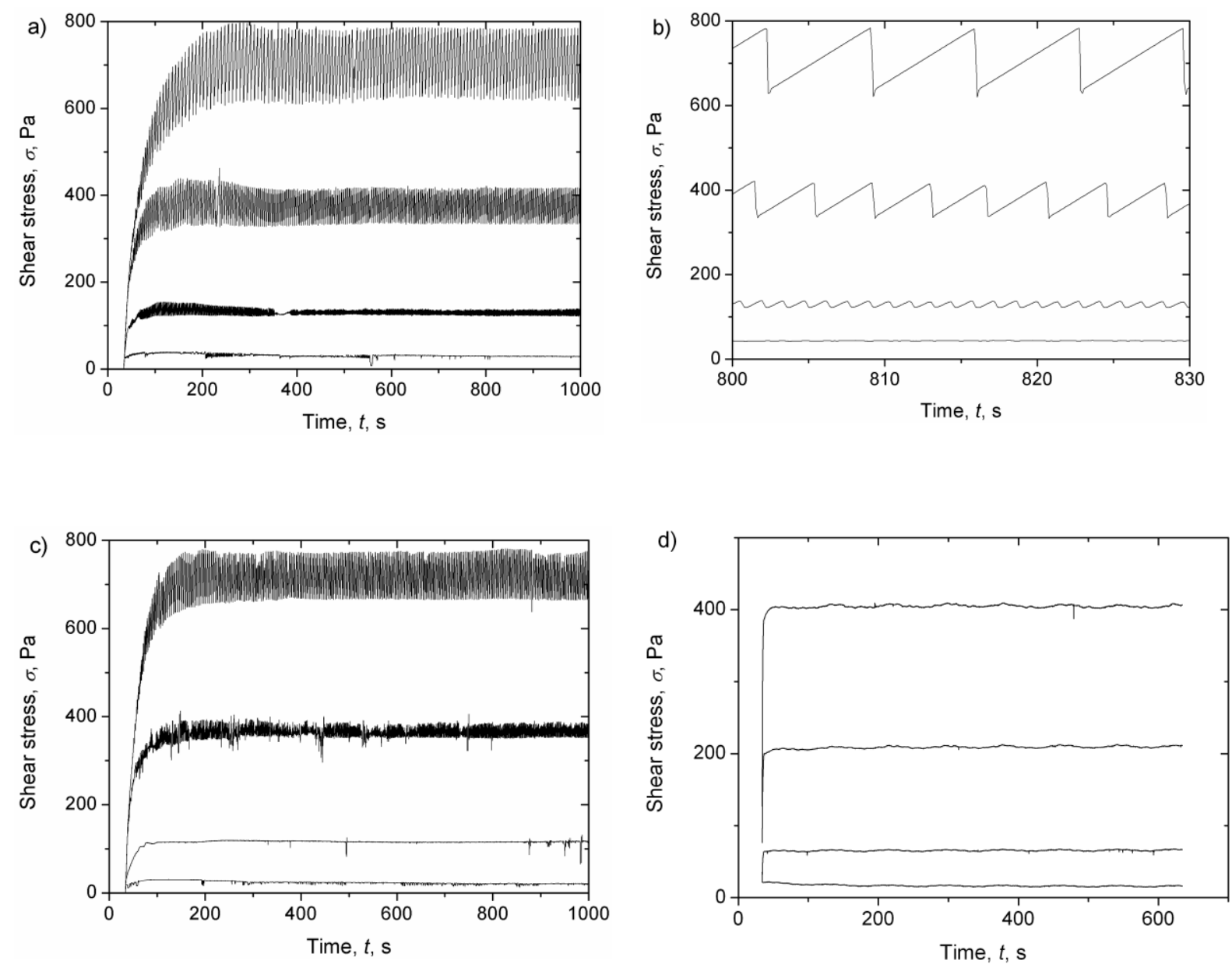

FIG.4. Examples of the time dependence of the shear stress at a constant shear rate, $\dot{\gamma}$, developed by highly concentrated MR suspensions upon application of a magnetic field, $H_{0}$. (a) $\dot{\gamma}=0.01 \mathrm{~s}^{-1}$; (b) the same as (a) but with an enlarged time scale; (c) $\dot{\gamma}=0.015 \mathrm{~s}^{-1}$; (d) $\dot{\gamma}=1 \mathrm{~s}^{-1}$. The four curves in each graph correspond to the applied magnetic fields increasing progressively from the lower to the upper curve and taking the following values: $0 ; 9 ; 18$ and $26.5 \mathrm{kA} / \mathrm{m}$.

Qualitatively, the period of oscillations increases when the magnetic field is increased and decreases with an increasing shear rate. At high fields and low shear rates, the slip time, $t_{\text {slip }}$, is an order of magnitude smaller than the stick time, $t_{\text {stick }}$ (for instance, $t_{\text {slip }}=0.10 \pm 0.05 \mathrm{~s}$ and $t_{\text {stick }}=6.6 \pm 0.1 \mathrm{~s}$ at $\dot{\gamma}=0.01 \mathrm{~s}^{-1}$ and $H_{0}=26.5 \mathrm{kA} / \mathrm{m}$, cf. Fig. 4b). On other hand, with decreasing magnetic fields or increasing shear rates, the amplitude of the stress oscillations 
decreases in value and the slip time turns out to be comparable to the stick time (as in the case of $\dot{\gamma}=0.01 \mathrm{~s}^{-1}$ and $H_{0}=9 \mathrm{kA} / \mathrm{m}$, see Fig. $4 \mathrm{~b}$ ). Finally, for shear rates higher than some critical value (for instance, $0.025 \mathrm{~s}^{-1}$ at $H_{0}=26.5 \mathrm{kA} / \mathrm{m}$ ) the regular stick-slip motion disappears and only small stress oscillations (with amplitude smaller than $2 \%$ of the mean stress value) are observed, as illustrated in Fig. $4 \mathrm{~d}$ for $\dot{\gamma}=1 \mathrm{~s}^{-1}$. These small oscillations are attributed to the instrumental error, of the order of $5 \%$, caused by a non-perfectly symmetric filling of the rheometer gap and eventual misalignment of the measuring geometry. The absence of regular oscillations, along with the absence of a clear minimum of the flow curve at zero field, allows us to conclude that the stick slip phenomenon may only appear in the presence of the applied magnetic field.

The effect of the applied magnetic field on the stick-slip behavior of the MR suspension is better illustrated in Fig. 5, where the period and amplitude (peak-to-peak) for a shear rate $\dot{\gamma}=0.01 \mathrm{~s}^{-1}$ (Fig. 5a), as well as the critical shear rate at which the oscillations disappear (Fig. 5b), are plotted as a function of the applied field strength. All these quantities increase progressively with the magnetic field. The increasing trend of the amplitude of oscillations and of the critical shear rate is an expected behavior, explained by the strengthened magnetic interactions between the particles, leading to more robust structures, which better withstand the applied shearing forces. The increasing tendency of the period of oscillation will be discussed at the end of the present section along with the microscopic interpretation of the stick-slip. Note also that the critical shear rates at which the stick-slip oscillations disappear are lower than the shear rate values corresponding to the minima of the flow curves presented in Fig. 3. This is probably because the flow curves have deep but rather flat minimum, around which the shear stress varies only slightly, such that pronounced instabilities do not occur in the vicinity of the minimum. 

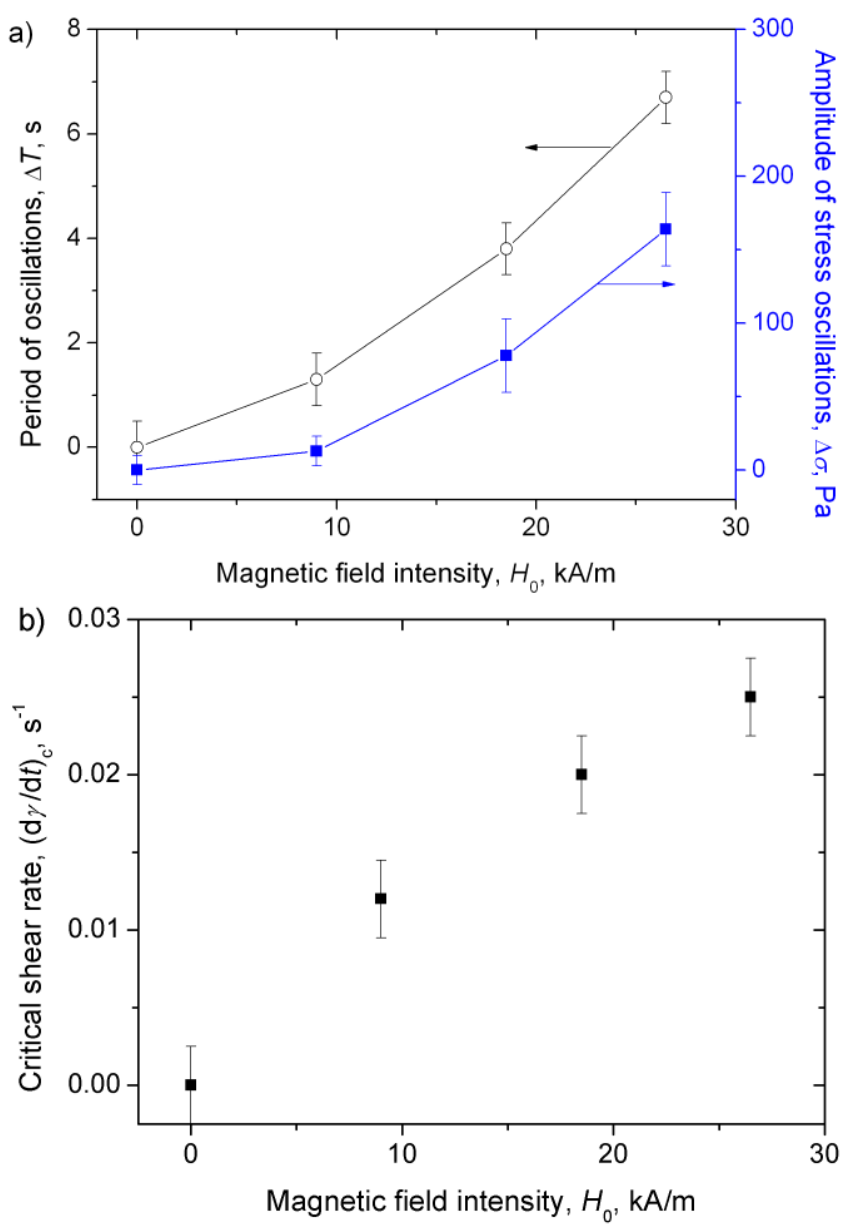

FIG. 5. (a) Period and amplitude of oscillations plotted as a function of the applied field strength for a shear rate of $0.01 \mathrm{~s}^{-1}$. (b) Field dependency of the critical shear rate, at which stick-slip disappears.

If we want to have a clear idea about what happens to the particle structures and/or to the shear field during stress oscillations, some visualization experiments coupled with macroscopic measurements should be performed. Most of the methods successfully used in conventional suspensions (dynamic light scattering, particle image velocimetry, magnetic resonance imaging, neutron or X-ray scattering) are not at all suitable for MR suspensions, due to their opacity, large particle size and the use of external magnetic fields. Therefore, we were forced to use the simplest method, namely, the direct visualization of the shear field of the MR sample by looking at the deformation of a pigmented column introduced into the meniscus of the MR suspension, as shown in Fig. 6. In more details, the pigmented column 
was a concentrated suspension of titanium dioxide nanoparticles in glycerol, and we proceeded as follows. After having placed the MR sample in the measuring geometry of the rheometer and pre-sheared it, we scratched a small canal on the meniscus surface of the MR sample in the direction perpendicular to the rheometer plates - this scratch was done in the presence of the magnetic field. Then, by using a micro-needle we filled the canal with the pigmented suspension, taking care for full filling of the canal. Finally, the sample was sheared at constant rate. A similar procedure was used by Persello et al. (1994) and Pignon et al. (1996) in their studies on the rheology of silica gels and laponite clays. Of course, this method does not allow inspection of the internal structure of the MR suspension and gives only a qualitative picture of the local shear profiles.
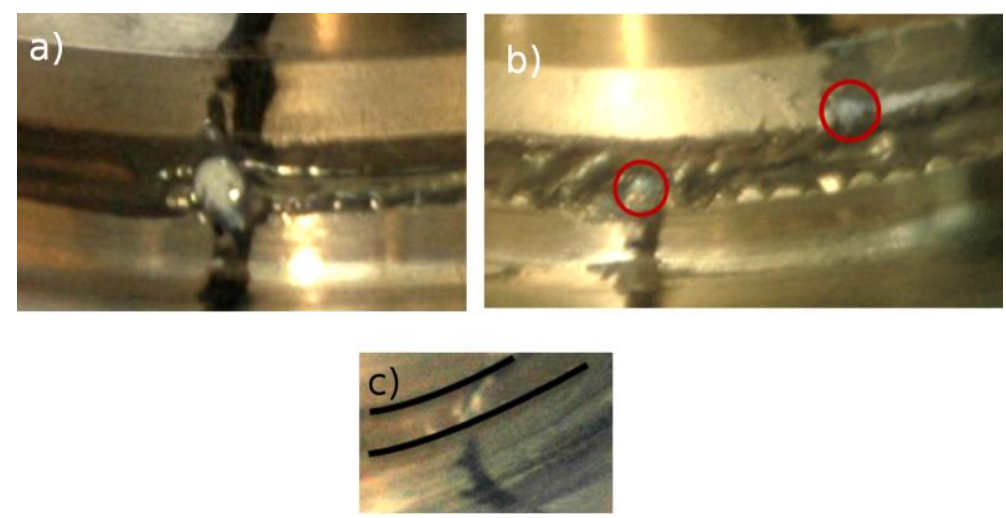

FIG. 6. Direct visualization of shear localization in highly concentrated MR suspensions upon application of a magnetic field strength, $H_{0}=18.5 \mathrm{kA} / \mathrm{m}$. At subcritical shear rate, $\dot{\gamma}=0.01 \mathrm{~s}^{-1}$, the initially straight pigmented column (a) breaks into two parts in the middle plane, the lower part being adhered to the lower plate and the upper part moving as a whole with the upper plate (b). This behavior could be interpreted in terms of shear localization at the middle plane. At supercritical shear rate, $\dot{\gamma}=0.5 \mathrm{~s}^{-1}$, the column is strained in an affine way (c), manifesting a homogeneous deformation of the suspension. The red circles in (b) surround the two parts of the broken pigmented column. The black curves in (c) are guidelines indicating the position of the upper and lower rheometer plates.

As observed in Figs. $6 a$ and $6 \mathrm{~b}$, at low shear rate, $\dot{\gamma}=0.01 \mathrm{~s}^{-1}$, for which regular stickslip oscillations were detected, the pigmented column was ruptured approximately at its 
middle height, the lower half remaining attached to the immobile lower plate, while the upper half moving jointly with the upper plate. Apparently, during the motion of the upper plate, both parts of the column seemed to be always perpendicular to the rheometer plates. At higher shear rate, $\dot{\gamma}=0.5 \mathrm{~s}^{-1}$, for which the stick-slip instability did not occur, the pigmented column experienced a uniform deformation, spanning the gap between both plates and elongating as it was tilted by the shear (see Fig. 6c). This could be taken as a proof of the existence of a homogeneous deformation profile, corresponding to a stable steady-state flow, at supercritical shear rates. On the other hand, the rupture of the pigmented column at subcritical shear rates could be interpreted in terms of suspension fracture in the middle plane, with shear localization occurring within a narrow lubrication layer.

In order to explain the stress oscillations on the basis of the existence of a narrow lubrication layer, we must assume that this layer periodically appeared and disappeared, the following being a likely scenario. The slow stress growth during the stick process could be attributed to a homogeneous elastic deformation of the suspension as a whole. At some critical strain, the suspension structure broke in the middle plane and the resulting blocks (each of the two halves of the suspension) turned back, sliding over each other, which provoked a rapid stress release. A scheme of such behavior is shown in Fig. 7. 


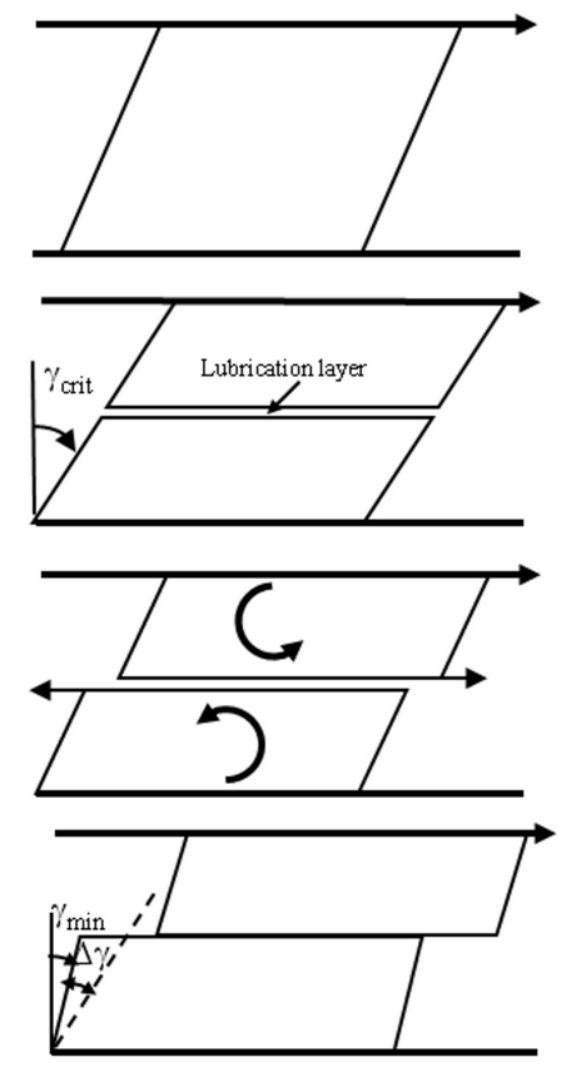

FIG. 7. Macroscopic description of the different stages of the stick-slip phenomenon. From top to bottom: homogeneous deformation of the suspension as a whole; fracture of the suspension structure in the middle plane at a critical strain, $\gamma_{\text {crit }}$ (onset of the slip with formation of a lubrication layer); backward rotation of the two suspension blocks with a sliding friction between them; stick of two blocks at some minimum strain, $\gamma_{\min }$. In the bottom sketch it is indicated that during the block rotation (slip process) the local strain decreases by $\Delta \gamma$.

Since we found experimentally that the stress did not release to zero but was lowered by a maximum of thirty percent (see Fig. 4), it was likely that the two suspension blocks did not recover initial zero strain but turned back to some small angle at which they stuck to each other once again. Thus, the local strain of both suspension blocks oscillated between some maximum value corresponding to the onset of the slip and some minimum value corresponding to the onset of the stick. The spatial period, $\Delta \gamma$, of these oscillations can be expressed in terms of the local strain of the suspension blocks; $\Delta \gamma$ is related to the time period, $\Delta \mathrm{T}$, by the formula $\Delta \gamma=\dot{\gamma}_{\text {glob }} \cdot \Delta \mathrm{T}$, where $\dot{\gamma}_{\text {glob }}$ is the global shear rate. Following this 
reasoning, we estimate the spatial period, $\Delta \gamma$, to be of the order of 0.035 at $H_{0}=18.5 \mathrm{kA} / \mathrm{m}$ and 0.06 at $H_{0}=26.5 \mathrm{kA} / \mathrm{m}$. These values correspond to the displacements of the upper rheometer plate by a distance of a few particle diameters, $2 a$. So, $\Delta \gamma$, is of the order of the ratio, $2 a / h$, of the particle diameter to the gap thickness, $h$; namely, $\Delta \gamma \approx 2.5 \cdot(2 a) / h$ at $H_{0}=18.5 \mathrm{kA} / \mathrm{m}$ and $\Delta \gamma \approx 4(2 a) / h$ at $H_{0}=26.5 \mathrm{kA} / \mathrm{m}$. The smallness of the strain $\Delta \gamma$ suggests that the stick-slip phenomenon occurs at the particle scale, i.e. when the two suspension blocks slide over each other a distance of the order of magnitude of the particle diameter (cf. Fig.7). The field effect on the spatial period and the time period of oscillations [Fig. 5a] could be explained by the formation of thicker particle structures with a larger spatial period when the magnetic field is increased. Such a behavior (the formation of thicker particle structures as the applied field is increased) has recently been revealed by X-ray microtomography [Borbáth et al. (2012)].

In next section, we present a theoretical model based on the considered microscopic mechanism of the stick-slip phenomenon, and we estimate the period and amplitude of the stress oscillations.

\section{THEORY AND DISCUSSION}

As already stated, the most likely mechanism that may cause the stick-slip phenomenon in MR suspensions is the periodic rupture and reformation of the particle aggregates. In a first approximation, the field-induced structure of a concentrated MR suspension can be presented as a periodic array of column-like aggregates that are often reported in literature [see, for instance, Borbáth et al. (2012)]. In more details, we may consider a hexagonal array of multi-chain aggregates whose thickness (or, equivalently, the number of single chains per one multi-chain) increases with the magnetic field. At this stage, 
we do not attempt to predict the aggregate thickness as function of the applied field. Instead, we restrict our theoretical analysis to the case of an applied magnetic field, $H_{0}=18.5 \mathrm{kA} / \mathrm{m}$, keeping in mind that our model could be applied to any value of the applied field just by using the correct spatial period. At a chosen magnetic field, $H_{0}=18.5 \mathrm{kA} / \mathrm{m}$, the spatial period found from the temporal period of stress oscillations is equal to $\Delta \gamma \approx 2.5(2 a) / h$ and appears to be close to the period, $\Delta \gamma \approx 1.9(2 a) / h$ of the hexagonal array of three-chain aggregates shown in Fig.1c. In what follows, we will estimate the principal parameters of the stick-slip motion for this particular structure.

During the stick process, the multi-chains are supposed to be strained in an affine way with the whole suspension, and the strain is supposed to grow linearly with time. The multichains are tilted by the applied strain and the suspension stress rises monotonically with the strain and, consequently, with the time. However, at some critical strain, the stress achieves its maximum value and then decreases with the strain. As discussed in one of our previous work [López-López et al. (2012)], at strains higher than the critical one the MR structure becomes mechanically unstable and, thus, it is supposed to be ruptured at the maximum point of the stress-strain relation. The critical strain was estimated to be $\gamma_{\text {crit }}=0.115$, and the failure of the MR structure at $\gamma_{\text {crit }}=0.115$ likely corresponds to the onset of the slip. According to our visualization experiments (see Fig. 6), the multi-chain aggregates are expected to be ruptured in their middle point. Afterwards, during the slip process, the upper and lower halves of the ruptured aggregates are supposed to turn back until they reach the opposite halves of the neighboring aggregates, as schematically depicted in Fig. 1c. Once in front of each other, due to the attractive magnetic forces, the aggregate halves are supposed to stick immediately to each other; this gives rise to the onset of the stick process. Thus, during the stick-slip process, the aggregates are supposed to perform angular oscillations with amplitude 
$\Delta \theta \approx \Delta \gamma \approx 1.9 \cdot(2 a) / h$, such that the local strain (associated to the angle $\theta$ between the aggregates and the magnetic field) fluctuates between the maximum value $\gamma_{\text {crit }} \approx 0.115$ and the minimum value $\gamma_{\min }=\gamma_{c r i t}-\Delta \gamma$ (see Fig. 7).

The temporal variation of the local strain during the slip can be estimated by equating the magnetic and hydrodynamic torques acting on the network of half-chains. Because of the symmetry of the problem with respect to the middle plane, the motion of both the lower and the upper networks is completely equivalent, so that we may consider only one of the networks. We assume that the aggregates move only in the shear plane and, consequently, their rotation axis is parallel to the direction of the vorticity. The magnetic torque per unit volume of the network can be found, in the general case, as the derivative of the network free energy (or, equivalently, of the thermodynamic function $\tilde{F}$ ) with respect to the strain angle $\theta$.

$$
T_{m}=\left(\frac{\partial \tilde{F}}{\partial \theta}\right)_{T, \mathbf{H}}
$$

The thermodynamic function $\tilde{F}$ is defined through the free energy of the suspension per unit volume, $F_{0}$, and can be written in terms of the local strain angle $\theta$ as follows [López-López et al. (2012)]:

$$
\tilde{F}=F_{0}-\frac{1}{2} \mu_{0} \mu_{\|} H^{2} \cos ^{2} \theta-\frac{1}{2} \mu_{0} \mu_{\perp} H^{2} \sin ^{2} \theta
$$

where $\mu_{0}=4 \pi \cdot 10^{-7} \mathrm{H} / \mathrm{m}$ is the magnetic permeability of vacuum, $\mu_{\|}$and $\mu_{\perp}$ are the components of the magnetic permeability tensor of the suspension in the reference frame of the aggregates and along their major and minor axes, respectively, $H$ is the magnetic field intensity inside the MR fluid. Writing down the last equation, we have supposed that the 
suspension magnetic permeability is field-independent; this assumption is verified by the magnetization measurements [cf. Fig.2] for the experimental range of the magnetic fields (0$26.5 \mathrm{kA} / \mathrm{m}$ ). By substitution of Eq. (2) into Eq. (1), the following expression is obtained for the magnetic torque:

$$
T_{m}=\mu_{0} H^{2}\left(\mu_{\|}-\mu_{\perp}\right) \sin \theta \cos \theta-\frac{1}{2} \mu_{0} H^{2}\left[\frac{\partial \mu_{\|}}{\partial \theta} \cos ^{2} \theta+\frac{\partial \mu_{\perp}}{\partial \theta} \sin ^{2} \theta\right] .
$$

The components, $\mu_{\|}$and $\mu_{\perp}$ of the magnetic permeability tensor, were calculated elsewhere [López-López et al. (2012)] for a multi-chain structure. This structure was designed in such a way that it could expand without losing the mechanical equilibrium until a critical strain at which it broke. In Eq. (3) the derivatives of the longitudinal and transverse magnetic permeabilities of the suspension are calculated as $\partial \mu_{k} / \partial \theta=\left(\partial \mu_{k} / \partial \gamma\right) /(\partial \gamma / \partial \theta)=\left(\partial \mu_{k} / \partial \gamma\right) \cdot\left(1+\gamma^{2}\right), \quad k=\|, \perp$, where the magnitudes $\partial \mu_{\|} / \partial \gamma$ and $\partial \mu_{\perp} / \partial \gamma$ are given in our previous work [López-López et al. (2012)]. Note that the transverse permeability appears to be almost constant, such that $\partial \mu_{\perp} / \partial \gamma \approx 0$. Taking into account the relation between the strain and the strain angle, $\gamma=\tan \theta$, the magnetic torque can be expressed in terms of the local strain:

$$
T_{m}=\mu_{0} H^{2}\left(\mu_{\|}-\mu_{\perp}\right) \frac{\gamma}{1+\gamma^{2}}-\frac{1}{2} \mu_{0} H^{2}\left[\frac{\partial \mu_{\|}}{\partial \gamma}+\frac{\partial \mu_{\perp}}{\partial \gamma} \cdot \gamma^{2}\right]
$$

According to [López-López et al. (2012)], $\mu_{\|}-\mu_{\perp} \ll\left|\partial \mu_{\|} / \partial \gamma\right|$, therefore, the first term of Eq.(4) appears to be negligible with respect to the second one and can be omitted. Note that, because of the demagnetizing field of the MR sample, the magnetic field intensity $H$ inside the suspension is lower than the intensity $H_{0}$ of the external field by a factor equal to the 
component, $\mu_{z z}$, of the magnetic permeability tensor of the suspension [López-López et al. (2012:

$$
H=\frac{H_{0}}{\mu_{z z}}=\frac{1+\gamma^{2}}{\mu_{\|}+\mu_{\perp} \gamma^{2}} H_{0} .
$$

The hydrodynamic torque acting on the network of half-chains can be estimated assuming that the network rotates in the quiescent ambient liquid, in such a way that in the reference frame of the aggregates the liquid filtrates through the interstices between them. It is clear that in its rotation the network will partially drag the suspending liquid. Therefore, since the hydrodynamic torque depends on the relative velocity of the network with respect to the liquid, the assumption of the quiescent liquid is a rough approximation to the problem, which will, nevertheless, allow us to estimate the order of magnitude of the slip time. The torque will be calculated using some kind of slender body theory adapted to an array of parallel slender aggregates. The normal component of the hydrodynamic force, $f_{\perp}$, acting per unit height of the network can be related to the hydraulic permeability of the network via the Darcy filtration law. In the Appendix, we demonstrate that $f_{\perp}$ is expressed through the velocity, $v_{\perp}=\dot{\theta} z$, of the multi-chain at its longitudinal position $z$ by the following formula:

$$
f_{\perp}=\frac{\eta_{0} v_{\perp} S}{r_{a, h}^{2} K}=\frac{\eta_{0} S}{2 a^{2} K} \dot{\theta} z
$$

where $\eta_{0}$ is the viscosity of the suspending liquid of the MR suspension, $S$ is the surface of the rheometer plates, $r_{a, h}=a \sqrt{2}$ is the hydraulic radius of the three-chain aggregates, $K$ is the dimensionless hydraulic permeability of the hexagonal array of cylindrical aggregates (given in Appendix), and $\dot{\theta}$ is the angular velocity of the half-chains. Now, the hydrodynamic torque per unit volume of network is calculated as the integral of the product $\left(f_{\perp} z\right)$ along the height 
of the network, $h / 2$, in a similar way as in the classical slender body theory [Batchelor $(1970,1971)]:$

$$
T_{h}=\frac{1}{V} \int_{0}^{h / 2} f_{\perp} z d z .
$$

Replacing the force density $f_{\perp}$ by Eq. (6), taking into account that $\dot{\theta} \approx \dot{\gamma}$ at small angles $\theta$, and noting that the ratio of the surface to the volume, $S / V$, is equal to the inverse of the height of the network, 2/h, Eq. (7) takes the following form:

$$
T_{h} \approx \frac{\eta_{0}(h / a)^{2}}{24 K} \dot{\gamma}
$$

where $\dot{\gamma} \neq \dot{\gamma}_{\text {glob }}$ is the local shear rate associated to the structure rotation. Note that the eventual compression of the aggregates during their rotation, something which decreases their height, $h / 2$, is an effect of second order of smallness on $\theta$ and, therefore, is not taken into account in our work. In the inertialess limit, the sum of both the magnetic and the hydrodynamic torques, Eqs. (4) and (8), must be equal to zero, which gives a first-order differential equation with respect to the local strain $\gamma$. This equation admits an approximate solution if the magnetic torque is interpolated by the following linear function of the strain:

$$
T_{m} \approx c \cdot(1 / 2) \mu_{0} H^{2} \cdot \gamma,
$$

with $c \approx 360$ being a numerical constant. At small variations of the strain, $\Delta \gamma \ll \gamma_{c r i t}$, the strain is found to decrease quasi-linearly with the time according to the equation:

$$
\gamma=\gamma_{c r i t} \exp \left(-\frac{t}{\tau}\right) \approx \gamma_{c r i t}\left(1-\frac{t}{\tau}\right)
$$


where $\gamma_{\text {crit }} \approx 0.115$ is the strain at the onset of the slip, and $\tau$ is the relaxation time given by:

$$
\tau=\frac{\eta_{0}(h / a)^{2}}{12 c \cdot K \mu_{0} H^{2}},
$$

with the internal magnetic field intensity $H(\gamma)$ corresponding to the critical strain, $\gamma_{c r i t}$. This time is the characteristic time of the structure rotation under the action of the magnetic torque. As observed in Eq. (11), the relaxation time is proportional to the viscosity of the suspending liquid and inversely proportional to the square of the magnetic field intensity $H$ and to the hydraulic permeability of the network. The slip time is simply calculated from Eq. (10) as the time required to decrease the strain by the value $\Delta \gamma \approx 1.9 \cdot(2 a) / h$ :

$$
t_{\text {slip }} \approx \frac{\Delta \gamma}{\gamma_{\text {crit }}} \tau
$$

For the magnetic field, $H_{0}=18.5 \mathrm{kA} / \mathrm{m}$, Eqs. (11) and (12) give, respectively, a relaxation time $\tau \approx 1.21 \mathrm{~s}$ and a slip time $t_{\text {slip }} \approx 0.29 \mathrm{~s}$. The latter is of the same order of magnitude than the experimental value $(0.20 \pm 0.05 \mathrm{~s}$, cf. Fig. 4b). The discrepancy is likely related to the assumption of quiescent suspending liquid, an assumption that overestimates the hydrodynamic dissipation and leads to a higher slip time than the one observed in the experiments. The complete stick-slip cycle is constituted by a linear growth of the local strain during the stick time and a quasi-linear decrease of the local strain during the slip time, according to the following equation:

$$
\gamma=\left\{\begin{array}{l}
\gamma_{\min }+\dot{\gamma}_{\text {glob }} t, \quad 0 \leq t \leq t_{\text {stick }} \\
\gamma_{\text {crit }}\left[1-\left(t-t_{\text {stick }}\right) / \tau\right], \quad t_{\text {stick }} \leq t \leq \Delta T
\end{array},\right.
$$

where $\Delta T=t_{\text {stick }}+t_{\text {slip }}$ is the total period of the stick-slip cycle. The time response of the suspension shear stress during the stick-slip cycle is simply calculated by substituting Eq. (13) 
in the expression for the magnetostatic stress. This expression was obtained in our previous work for highly concentrated MR suspensions [López-López et al. (2012)]:

$$
\sigma=\mu_{0} H^{2}\left(\mu_{\|}-\mu_{\perp}\right) \frac{\gamma}{\left(1+\gamma^{2}\right)^{2}}-\frac{1}{2} \mu_{0} H^{2}\left[\frac{\partial \mu_{\|}}{\partial \gamma} \cdot \frac{1}{1+\gamma^{2}}+\frac{\partial \mu_{\perp}}{\partial \gamma} \cdot \frac{\gamma^{2}}{1+\gamma^{2}}\right]+\frac{1}{2} \mu_{0} H^{2}\left(\mu_{\|}-\mu_{\perp}\right) \frac{\gamma}{1+\gamma^{2}}
$$

The theoretical time-dependencies of the local strain and the stress are plotted in Fig.8. The linear growth/decrease of the local strain is well illustrated in this figure, in accordance with Eq. (13), while the stress signal has a smoother shape. The latter is connected to the rounded shape of the stress-vs.-strain curve near its summit, where the structure breaks -see López-López et al. (2012). Because of this, when the critical strain is approached, the stress increases with the strain slower than linearly and, since the strain increases linearly with time during stick, the theoretical stress presents a less rapid increase. In fact, from the theoretical point of view $\partial \sigma / \partial \gamma \rightarrow 0$ when $\gamma \rightarrow \gamma_{\text {crit }}$. The theoretical stress signal (Fig. 8) qualitatively predicts the features of the experimental one (Fig. 4b). Both in theory and in experiments, we observe a monotonic stress growth during stick and a monotonic decrease during slip; the stress amplitude is an order of magnitude lower than the stress maximum; and the stick time is an order of magnitude higher than the slip one. However, the stress growth section is quasilinear in the experimental case, whereas it presents a rounded-shape curvature in the theoretical one. This discrepancy could be explained by the fact that the real stress versus strain curve has probably a sharper pick at the critical strain. 


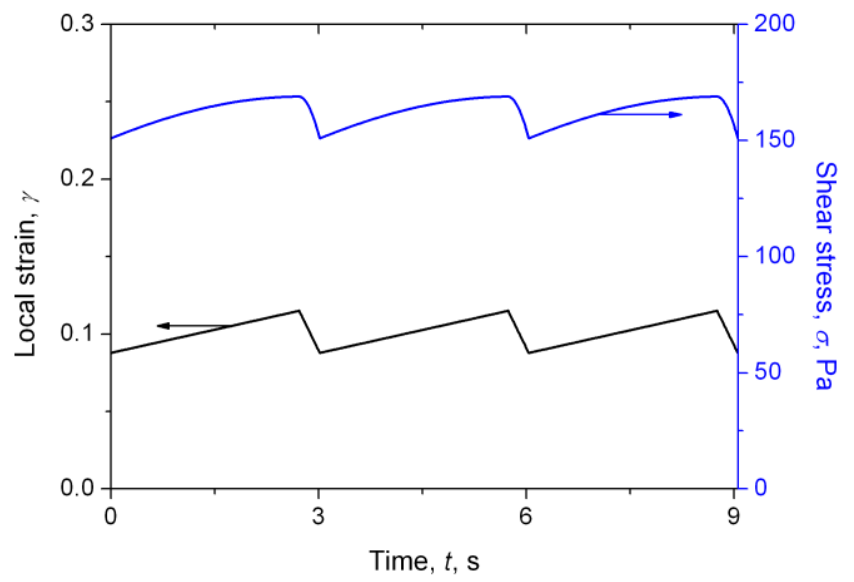

FIG. 8. Theoretical time dependencies of the local strain and of the suspension shear stress for a highly concentrated MR suspension upon application of a magnetic field of strength $H_{0}=18.5 \mathrm{kA} / \mathrm{m}$, and at the global shear rate $\dot{\gamma}_{\text {glob }}=0.01 \mathrm{~s}^{-1}$.

In what concerns the field dependency of the amplitude of stress oscillations, our model predicts an increasing trend in qualitative agreement with experiments (cf. Fig. 5a). In more details, the stress amplitude is given by the difference between the maximum stress, $\sigma_{\max }=\sigma\left(\gamma_{\text {crit }}\right)$, and the stress corresponding to the minimum strain $\gamma_{\min }=\gamma_{\text {crit }}-\Delta \gamma$. In the limit $\Delta \gamma<0.3 \gamma_{\text {crit }}$, the stress amplitude can be expanded into power series on $\Delta \gamma$, as follows: $\Delta \sigma=\sigma\left(\gamma_{\text {crit }}\right)-\sigma\left(\gamma_{\text {crit }}-\Delta \gamma\right) \approx-(1 / 2)\left(\partial^{2} \sigma / \partial \gamma^{2}\right)_{\gamma_{\text {crit }}}(\Delta \gamma)^{2}$, taking into account that $(\partial \sigma / \partial \gamma)_{\gamma_{c r i t}}=0$. Thus, deriving two times the shear stress [Eq. (14)] with respect to the strain, it becomes clear that the stress amplitude, $\Delta \sigma$, scales as $\mu_{0} H^{2}(\Delta \gamma)^{2}$, and therefore increases progressively with the magnetic field, taking into account that $\Delta \gamma$ is an increasing function of the field.

At this point is worth mentioning some related results of numerical simulations performed by other authors. Klingenberg et al. (1991) reported quite irregular oscillations of the stress in very dilute ER suspensions, for which collisions between broken chains are rather 
seldom and random events. Bonnecaze and Brady (1992) found quite irregular fluctuations of the electrostatic stress of electrorheological fluids at very low shear rates (Mason number $\left.M a=10^{-4}\right)$, which became more regular at higher shear rates $\left(M a=10^{-2}\right)$. Nevertheless, in the latter case, microscopic rearrangements of the particles inside the aggregates and between them seem to play an important role and provoke a non-negligible noise in the stress signal. Furthermore, the shape of the stress signal appears to be rather different from that observed in our experiments, with the slip time being approximately equal to the stick time and the amplitude of the stress oscillations comparable to the pick value of the stress. Such discrepancy with experiments could come from the fact that only a small number of particles was used in the simulations and, consequently, the influence of local stochastic events did not vanish.

Another important feature observed in our experiments is the disappearance of the stick-slip phenomenon at some critical shear rate - see Fig. 5b. At this moment, we do not have a clear explanation for the absence of the stick-slip at higher shear rates. We suppose that this could be connected to the kinetics of reformation of structures after their rupture at a critical (yield) stress.

A further analysis of the suspension microstructure inherent to the stick-slip instability requires knowledge of the interparticle gaps. Using our model of homogeneous elongation of the aggregates upon shearing, we estimate that, at the maximum extension, the interparticle gap is about $0.7 \%$ of the particle diameter, i.e. $33 \mathrm{~nm}$ for the particles with $5 \mu \mathrm{m}$ of diameter. Such a small gap is, at the most, of the same order of magnitude as the particle roughness. Therefore, we expect that solid friction between particles plays an important role in the reformation of aggregates during their backward motion. Once in contact, boundary lubrication between particle surfaces likely occurs, something which would induce high 
friction forces with features similar to those of solid friction [Persson (2000)]. In order to check if the contacts between particles are governed by hydrodynamic lubrication (squeeze repulsive forces) or boundary lubrication (friction forces), we calculated the ratio of squeeze forces to normal interparticle forces:

$$
\frac{F_{s}}{F_{n}}=\frac{3 \pi \eta_{0} a^{2} v /(2 \varepsilon)}{\pi a^{2} f_{m}} \sim \frac{\eta_{0} v}{f_{m} \varepsilon},
$$

where $v \sim \Delta \gamma h / t_{\text {slip }}$ is the characteristic velocity of approach of particles belonging to opposite aggregates, $\varepsilon \sim 50 \mathrm{~nm}$ is the particle roughness and $f_{m}$ is the characteristic magnetic force (per unit particle cross-section, $\pi a^{2}$ ) between two particles separated by a distance $\varepsilon$. We calculated the force $f_{m}$ by integration of the Maxwell stress tensor over the outer face of the surface of one of the particles using finite element method simulations [software FEMM, Meeker (2009)]. We obtained that $f_{m} \approx 360 \cdot \mu_{0} H^{2}$, with $H$ being the magnetic field inside the MR fluid [cf. Eq. (5)] at the critical strain, $\gamma_{\text {crit }} \approx 0.115$. For the experimental parameters corresponding to the stick-slip phenomenon shown in Fig. 4 b, Eq. (15) gives $F_{S} / F_{n} \sim 10^{-2}$, which confirms the importance of friction forces for particles in contact. Thus, when two halves of the broken aggregates come into contact, a tangential friction force likely appears in the contact area, oriented in the direction of the applied shear. Estimations show that this force is relatively strong and creates a supplementary torque, which very likely balances the restoring magnetic torque and stops the backward rotation of the broken aggregates at the first encounter with the neighboring ones. Of course, friction should also affect the conditions of the structure failure [as pointed out by Tian et al (2010)] and could thus enhance the stress level in the suspension. However, this matter is out of scope of the present paper and will be considered in a future work. 


\section{CONCLUSIONS}

We have shown that the rheograms (shear stress-vs.-shear rate curves) of concentrated MR suspensions present a decreasing branch at low values of the shear rate. In this region of negative differential viscosity the stable flow of the suspension is impossible, as proved by the stress oscillations obtained for each imposed shear rate within this decreasing branch. The obtained stress oscillations present well-defined amplitude and period, which appear to increase with the applied field strength. These stress oscillations are reminiscent of the stickslip instabilities reported by other authors for different colloidal suspensions and, in fact, our macroscopic visualizations have shown that the observed oscillations are correlated with the appearance of a fracture layer at middle height of the sheared suspension. Consequently, we have explained these oscillations by a mechanism of elongation of the field-induced particle aggregates until their failure at a critical strain, followed by recombination among broken parts of neighboring aggregates. Taking this as the starting point, we have developed a model based on the balance of the torques acting on the field-induced particle structures. Our model predicts a magnetic field dependence characterized by an increase of the amplitude of the stress oscillations, explaining it in terms of enhanced magnetic interactions. Finally, we have tried to estimate whether the flow of our suspension corresponds to lubricated contacts between particles (squeeze repulsive regime) or to direct contacts with solid friction (frictional regime). Calculations have shown that friction forces dominate over squeeze forces for the characteristic parameters of our experiments. Nevertheless, the solid friction between particles is expected to be a supplementary but not decisive mechanism of the stick-slip phenomenon. We should maintain the rupture/reformation mechanism as the principle reason for the stick-slip instabilities observed in concentrated MR suspensions. 


\section{ACKNOWLEDGEMENTS}

We would like to thank Drs. Laurent Lobry and Andrey Zubarev for helpful discussions. Financial support by projects Fis2009-07321 (Ministerio de Ciencia e Innovación, Spain), P08-FQM-3993 and P09-FQM-4787 (Junta de Andalucía, Spain), and "Factories of the Future" (Grant No. 260073, DynExpert FP7) is gratefully acknowledged. MTL-L and LR-A acknowledge financial support by the University of Granada (Spain) and Secretaría de Estado de Educación, Formación Profesional y Universidades (MECD, Spain) through its FPU program respectively.

\section{APPENDIX: HYDRAULIC RESISTANCE OF THE NETWORK OF MULTI-CHAIN AGGREGATES}

Let us consider a laminar filtration flow of an interstitial liquid through a periodic hexagonal array of triple-chain aggregates, as shown in Fig. A1. The flow is perpendicular to the aggregates and the superficial velocity is equal to $v_{\perp}$. Our aim is to calculate the hydrodynamic force, $f_{\perp}$, acting per unit height, $d h$, of this network.

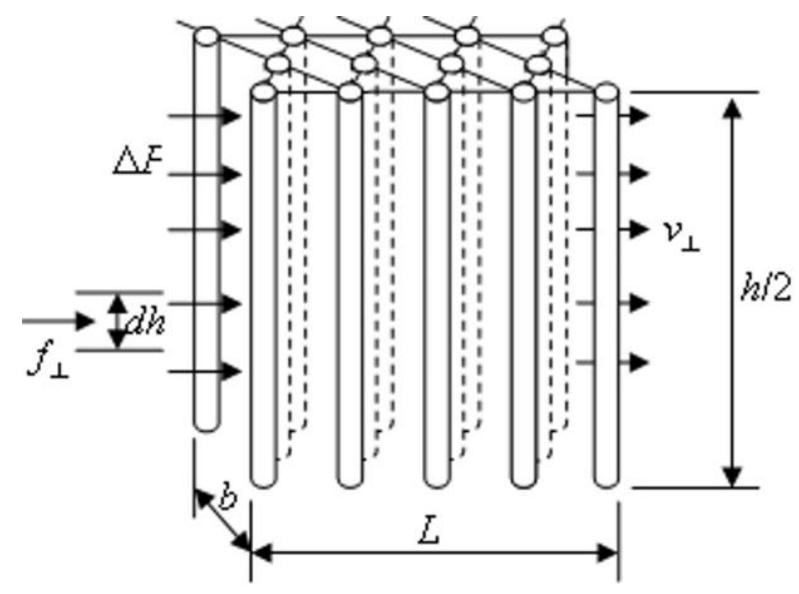

FIG. A1. Schematic representation of a hexagonal array of chain-like aggregates. 
The distance between chain the axes, given in Table II, is $d=2 \sqrt{\pi /(\sqrt{3} \Phi)} \cdot a \approx 1.9 \cdot(2 a)$. It is reasonable to approximate the elongated shape of the chains by a straight cylinder with equivalent (or hydraulic) radius corresponding to the radius of a cylindrical column having equal volume to that of the solid phase of the chain: $r_{a, h}=a \sqrt{2}$. The unit hydrodynamic force, $f_{\perp}$, can be expressed through the pressure difference, $\Delta P$, of the liquid across the network:

$$
f_{\perp}=\Delta P \cdot b
$$

where $b$ is the network dimension perpendicular to the flow (network width). The pressure difference is found from the Darcy filtration law with an appropriate hydraulic permeability, $K$, of the network:

$$
\Delta P=\frac{\eta_{0} L}{r_{a, h}{ }^{2} K} v_{\perp}
$$

where $L$ is the network dimension along the flow (network length) and the permeability $K$ is normalized by the square of the column radius, $r_{a, h}{ }^{2}$, and given by the expression derived by Bruschke and Advani (1993) using the lubrication approximation:

$$
K=\frac{\left(1-l^{2}\right)^{2}}{3 \sqrt{3} \cdot l^{3}}\left(3 l \frac{\operatorname{atan}(\sqrt{(1+l) /(1-l})}{\sqrt{1-l^{2}}}+\frac{1}{2} l^{2}+1\right)^{-1},
$$

with $l=2 r_{a, h} / d=\sqrt{2 \sqrt{3} \Phi / \pi} \approx 0.742$ being the ratio of the hydraulic diameter of the aggregates to the distance between them. By substitution of Eq. (A.2) into Eq. (A.1), and taking into account that the product $L b$ is the network surface perpendicular to the aggregates, $S$, we arrive to the final expression for the hydrodynamic force, $f_{\perp}$, which is given above by 
Eq. (6). Of course, in our experiments with shear flow in plate-plate geometry, we do not expect any pressure gradient along the circumferential direction of the flow. Filtration at homogeneous superficial velocity is considered here only for the purpose of the estimation of the network resistance coefficient. This coefficient (prefactor at $\eta_{0} v_{\perp}$ in the expression for $f_{\perp}$ ) is then used for the calculation of the hydrodynamic torque of the rotating network, Eqs. (7) and (8). The same artifice was used in the classical slender body theory for the calculation of the resistance coefficients for the Stokes flow around a cylinder, which were subsequently used for the calculation of the torque and the stresslet in shear flows [Batchelor (1970)].

\section{References}

Batchelor, G. K., "Slender-body theory for particles of arbitrary cross-section in Stokes flow," J. Fluid Mech. 44, 419-440 (1970).

Batchelor, G. K., "The stress generated in a non-dilute suspension of elongated particles by pure straining motion,” J. Fluid Mech. 46, 813-829 (1971).

Bécu, L., S. Manneville, and A. Colin, "Yielding and flowing adhesive and non-adhesive concentrated emulsions," Phys. Rev. Lett. 96, 138302 (2006).

Bonn, D., P. Coussot, H.T. Huynh, F. Bertrand, and G. Debrégeas, "Rheology of softglassy materials,” Europhys. Lett. 59, 786-792 (2002).

Bonnecaze, R. T., and J. F. Brady, "Dynamic simulation of an electrorheological fluid," J. Chem. Phys. 96, 2183-2202 (1992).

Borbáth T, S. Günther, D. Yu. Borin, Th. Gundermann, and S. Odenbach, "XmCT analysis of magnetic field-induced phase transitions in magnetorheological elastomers", Smart Mater. Struct. 21, 105018 (2012). 
Bruschke, M. V., and S. G. Advani, "Flow of generalized Newtonian fluids across a periodic array of cylinder," J. Rheol. 37, 479-498 (1993).

Coussot, P., J. S. Raynaud, F. Bertrand, P. Moucheront, J.P. Guilbaud, H.T. Huynh, S. Jarny, and D. Lesueur, "Coexistence of liquid and solid phases in flowing soft-glassy materials," Phys. Rev. Lett. 88, 218301 (2002).

Coussot, P., L. Tocquer, C. Lanos, and G. Ovarlez, "Macroscopic vs. local rheology of yield stress fluids,” J. Non-Newtonian Fluid Mech. 158, 85-90 (2009).

Da Cruz, F., F. Chevoir, D. Bonn, and P. Coussot, "Viscosity bifurcation in foams, emulsions and granular systems,” Phys. Rev. E 66, 051305 (2002).

de Vicente J. and J. Ramírez, "Effect of Friction between Particles in the Dynamic Response of Model Magnetic Structures,” J. Coll. Interface Sci. 316, 867-876 (2007)

Fall, A., F. Bertrand, G. Ovarlez, and D. Bonn, "Yield Stress and Shear Banding in Granular Suspensions,” Phys. Rev. Lett. 103, 17830 (2009).

Huang, N., G. Ovarlez, F. Bertrand, S. Rodts, P. Coussot, and D. Bonn, "Flow of wet granular materials," Phys. Rev. Lett. 94, 028301 (2005).

Ianni, F., R. Di Leonardo, S. Gentilini, and G. Ruocco, "Shear-banding phenomena and dynamical behavior in a Laponite suspension,” Phys. Rev. E 77, 031406 (2008).

Jarny, S., N. Roussel, S. Rodts, R. Le Roy, and P. Coussot, "Rheological behavior of cement pastes from MRI velocimetry,” Concrete Cement Res. 35, 1873-1881 (2005).

Jiang J., Yu Tian, and Y. Meng, "Stick-slip motion of MR fluids during shearing," in Abstracts of the 13th International Conference on Electrorheological Fluids and Magnetorheological Suspensions, ed. I. Unal (2012), pp. 138-139. 
Jiang J., Yu Tian, and Y. Meng, "Structure Parameter of Electrorheological Fluids in Shear Flow," Langmuir 27, 5814-5823 (2011)

Jiles, D., Introduction to Magnetism and Magnetic Materials (Chapman and Hill, London, 1991).

Klingenberg D. J., and C. F. Zukoski, "Studies on the steady-shear behavior of electrorheological suspensions," Langmuir 6, 15-24 (1990).

Klingenberg, D. J., F. Vanswol and C. F. Zukoski, "The small shear rate response of electrorheological suspensions .1. Simulation in the point-dipole limit," J. Chem. Phys. 94, 6160-6169 (1991).

Kuzhir P., M. T. López-López and G. Bossis, "Magnetorheology of fiber suspensions. II. Theory", J. Rheol. 53, 127-151 (2009).

Kuzhir, P., M. T. López-López, G. Vertelov, C. Pradille, and G. Bossis, “Shear and squeeze rheometry of suspensions of magnetic polymerized chains," Rheol. Acta 47, 179-187 (2008).

López-López, M.T., P. Kuzhir, J. Caballero-Hernández, L. Rodríguez-Arco, J. D. G. Durán, and G. Bossis, "Yield stress in magnetorheological suspensions near the limit of maximumpacking fraction,” J. Rheol. 56, 1209-1224 (2012).

Meeker D. C., Finite Element Method Magnetics, Version 4.2 (15 July 2009 Mathematica Build), http://www.femm.info

Ovarlez, G., F. Bertrand, and S. Rodts, "Local determination of the constitutive law of a dense suspension of non-colloidal particles through MRI," J. Rheol. 50, 259-292 (2006). 
Ovarlez, G., S. Rodts, X. Chateau, and P. Coussot, "Phenomenology and physical origin of shear localization and shear banding in complex fluids," Rheol. Acta 48, 831-844 (2009).

Persello, J., A. Magnin, J. Chang, J. M. Piau, B. Cabane, "Flow of colloidal aqueous silica dispersions," J. Rheol. 38, 1845-1870 (1994).

Persson, B. N. J., Sliding Friction. Physical principles and applications (Springer-Verlag, Berlin 2000).

Picard, G., A. Ajdari, L. Bocquet, and F. Lequeux, "Simple model for heterogeneous flows of yield stress fluids," Phys. Rev. E 66, 051501 (2002).

Pignon, F., A. Magnin, and J. M. Piau, “Thixotropic colloidal suspensions and flow curves with minimum: Identification of flow regimes and rheometric consequences," J. Rheol. 40, 573-587 (1996).

Quemada, D., “Unstable flows of concentrated suspensions,” Lect. Notes Phys. 164, 210-247 (1982).

Ragouilliaux, A., G. Ovarlez, N. Shahidzadeh-Bonn, B. Herzhaft, T. Palermo, and P. Coussot, "Transition from a simple yield stress fluid to a thixotropic material," Phys. Rev. E 76, 051408 (2007).

Rodts, S., J. C. Baudez, and P. Coussot, "From discrete to continuum flow in foams," Europhys. Lett. 69, 636-642 (2005).

Tian Yu, J. Jiang, Y. Meng, and Sh. Wen, “A shear thickening phenomenon in magnetic field controlled-dipolar suspensions,” Appl. Phys. Lett. 97, 151904 (2010-a) 
Tian Yu, M. Zhang, J. Jiang, N. Pesika, H. Zeng, J. Israelachvili, Y. Meng, and Sh. Wen, "Reversible shear thickening at low shear rates of electrorheological fluids under electric fields,” Phys. Rev. E 83, 011401 (2011)

Tian Yu, M. Zhang, X. Zhu, Y. Meng, and Sh. Wen, «Ultrahigh yield stress in a general electrorheological fluid under compression,” Smart Mater. Struct. 19, 035009(2010-b)

Varadan, P., and M. J. Solomon, "Direct visualization of flow-induced microstructure in dense colloidal gels by confocal laser scanning microscopy,” J. Rheol. 47, 943-968 (2003).

Woestman, J. T., “Stick-slip response in electrorheological fluids,” Phys. Rev. E 47, 29422945 (1993). 